BULLETIN OF THE

AMERICAN MATHEMATICAL SOCIETY

Volume 80, Number 6, November 1974

\title{
VARIETIES OF SMALL CODIMENSION IN PROJECTIVE SPACE ${ }^{1}$
}

\author{
BY ROBIN HARTSHORNE
}

Introduction. I would like to begin by stating a conjecture. While I am not convinced of the truth of this statement, I think it is useful to crystallize one's ideas, and to have a particular problem in mind. Then for the remainder of the talk, I propose to examine this question in a rather general way from a number of different perspectives. This will give me an opportunity to report on recent work in several areas of algebraic geometry, and at the same time to mention a number of open problems.

Let $k$ be an algebraically closed field. Let $\boldsymbol{P}^{n}$ be the $n$-dimensional projective space over $k$. Let $Y \subseteq \boldsymbol{P}^{n}$ be a nonsingular subvariety of dimension $r$. We say that $Y$ is a complete intersection in $\boldsymbol{P}^{n}$ if one can find $n-r$ hypersurfaces $H_{1}, \cdots, H_{n-r}$, such that $Y=H_{1} \cap \cdots \cap H_{n-r}$, and such that this intersection is transversal, i.e. the hypersurfaces $H_{i}$ are nonsingular at all points of $Y$, and their tangent hyperplanes intersect properly at each point of $Y$. In algebraic terms, $Y$ is a complete intersection if and only if its homogeneous prime ideal $I(Y) \subseteq k\left[x_{0}, \cdots, x_{n}\right]$ can be generated by exactly $n-r$ homogeneous polynomials.

Conjecture. If $Y$ is a nonsingular subvariety of dimension $r$ of $\boldsymbol{P}^{n}$, and if $r>\frac{2}{3} n$, then $Y$ is a complete intersection.

The paper is divided into six sections:

$\S 1$. Representing cohomology classes by subvarieties.

\$2. Cohomological properties of the subvariety.

§. Examples. Subvarieties of small degree.

$\S 4$. Embedding varieties in projective space.

$\S 5$. Connections with local algebra.

$\S 6$. Existence of vector bundles on $\boldsymbol{P}^{n}$.

1. Representing cohomology classes by subvarieties. To give perspective on our conjecture, let us consider some more general questions. Let $X$ be a nonsingular algebraic variety, and let $Y$ be a (possibly singular) subvariety. Then we can consider the cohomology class of $Y$, in any

An expanded version of an invited address delivered before the Missoula, Montana Meeting of the Society on August 24, 1973; received by the editors February 5, 1974.

AMS (MOS) subject classifications (1970). Primary 14C30, 14M10; Secondary $13 \mathrm{H} 10$.

Key words and phrases. Complete intersection, vector bundles, Gorenstein rings.

${ }^{1}$ Research supported by an NSF grant. 
suitable cohomology theory on $X$. To fix the ideas, let us suppose that $X$ is a projective variety defined over the complex numbers. Then we can consider $X$ as a compact complex manifold. If $Y$ is a subvariety, it defines a homology class on $X$, which by Poincaré duality gives us a cohomology class $\eta(Y) \in H^{2 \alpha}(X, Z)$, where $q$ is the (complex) codimension of $Y$ in $X$. This definition can be extended by linearity to give the cohomology class $\eta(Z)$ of any algebraic cycle $Z$ on $X$. An algebraic cycle of codimension $q$ on $X$ is by definition a formal linear combination $Z=\sum n_{i} Y_{i}$ of irreducible subvarieties $Y_{i}$ of codimension $q$ with integer coefficients $n_{i}$.

Once we have the notion of the cohomology class of a cycle, a number of questions come to mind:

Question 1.1. Which cohomology classes $\xi \in H^{2 q}(X, Z)$ are of the form $\eta(Z)$ for some algebraic cycle $Z$ ? If $\xi=\eta(Z)$ we say that $\xi$ is represented by the cycle $Z$.

Question 1.2. If $\xi$ can be represented by some algebraic cycle, can one find a cycle $Z=\sum n_{i} Y_{i}$ representing $\xi$, where the $Y_{i}$ are all nonsingular subvarieties?

Question 1.3. Which cohomology classes $\xi \in H^{2 q}(X, Z)$ can be represented by a single irreducible nonsingular subvariety $Y$ ?

Question 1.4. If $Y$ is a nonsingular subvariety of codimension $q$ in $X$, to what extent are properties of $Y$ determined by its cohomology class $\eta(Y)$ ?

Let me comment briefly on each of these questions.

For Question 1.1 there is a well-known necessary condition for $\xi$ to be representable, namely that the image of $\xi$ in $H^{2 q}(X, \boldsymbol{R})$ be representable by a differential form of type $(q, q)$ where $H^{*}(X, \boldsymbol{R})$ is calculated by the De Rham theorem using $C^{\infty}$ differential forms. Hodge [21] conjectured that this condition is also sufficient. Atiyah and Hirzebruch [2] showed that the Hodge conjecture in this strong form fails for torsion cohomology classes. This leaves open the "rational Hodge conjecture", which would say, for a class $\xi \in H^{2 q}(X, Z)$, that $n \xi$ can be represented by an algebraic cycle for some $n \neq 0, n \in Z$, if and only if the image of $\xi$ in $H^{2 \alpha}(X, R)$ is of type $(q, q)$.

Question 1.2 is essentially the question of "smoothing cycles for homological equivalence," which can be stated as follows. Given a cycle $Z=\sum n_{i} Y_{i}$ on $X$, can one find a cycle $Z^{\prime}=\sum n_{i}^{\prime} Y_{i}^{\prime}$ homologically equivalent to $Z$, i.e. with $\eta(Z)=\eta\left(Z^{\prime}\right)$, such that the subvarieties $Y_{i}^{\prime}$ are all nonsingular? This question has been studied by Hironaka [19] and Kleiman [24], who give affirmative answers in certain cases when the dimension of $Z$ is not too large with respect to the dimension of $X$. Recently Rees, Thomas, and I [16] have shown that the smoothing of cycles in this sense is not always possible. 
I do not know of any results in relation to Question 1.3 except for trivial cases such as $X=\boldsymbol{P}^{n}$. In that case $H^{2 q}(X, Z)$ is isomorphic to $Z$, with the cohomology class of a linear subspace of codimension $q$ serving as a generator. Under this isomorphism, an integer $d \in H^{2 q}(X, Z)$ is represented by a nonsingular subvariety if and only if $d>0$. For example, one can use a nonsingular hypersurface $Y$ of degree $d$ in $\boldsymbol{P}^{n-q+1} \subseteq \boldsymbol{P}^{n}$. This shows that for $X=\boldsymbol{P}^{n}$ the answers to Questions 1.1 and 1.2 are both trivially yes.

When the answer to Question 1.3 is known, then it is interesting to ask for more precise information about the possible nonsingular subvarieties representing a given cohomology class. As an illustration, let $X=\boldsymbol{P}^{3}$, and let $\xi \in H^{4}(X, Z)$ correspond to a positive integer $d$. Then for any nonsingular curve $Y$ of degree $d$ in $P^{3}$, we will have $\eta(Y)=\xi$. We can ask what are the possible values of the genus $g$ of $Y$. It is easy to give bounds on $g$ (for example $0 \leqq g \leqq \frac{1}{2}(d-1)(d-2)$, and both extremes are attained), but I do not believe that it is known exactly which values of $g$ can occur.

This should illustrate what I have in mind for Question 1.4. Now coming back to our conjecture, it can be viewed as a special case of Question 1.4. The general philosophy which operates here is that a nonsingular subvariety of small codimension of a fixed variety $X$ must be subject to stringent restrictions. More specifically, in the case of a subvariety $Y$ of $\boldsymbol{P}^{n}$, if we know that it is a complete intersection of hypersurfaces of degrees $d_{1}, \cdots, d_{n-r}$, then we essentially know all about it. We can calculate its cohomology groups, its fundamental group, and so on.

2. Cohomological properties of the subvariety. Another way we can approach our conjecture is this. The theorems of Lefschetz (which we will review in a minute) tell us a great deal about the cohomology of a complete intersection variety in projective space. We can ask to what extent do analogous properties hold for an arbitrary nonsingular subvariety. The remarkable recent results of Barth and others show that many of the same properties hold for nonsingular subvarieties of small codimension. This supports our conjecture in the sense that it shows that subvarieties of small codimension "look like" complete intersections from a cohomological point of view.

In this section I would like to review the Lefschetz theorems, and report on recent theorems of Barth type. I would also like to give a new proof of Barth's first theorem, by deriving it as a consequence of the "strong Lefschetz theorem". For simplicity, I will stick to varieties over $\boldsymbol{C}$.

THEOREM 2.1. Let $Y$ be a nonsingular subvariety of dimension $r$ of $\boldsymbol{P}_{C}^{n}$, which is a complete intersection. Then:

(a) The restriction map $H^{i}\left(\boldsymbol{P}^{n}, Z\right) \rightarrow H^{i}(Y, Z)$ is an isomorphism for $i<r$, and injective for $i=r$. 
(b) $\pi_{1}(Y)=1$ if $r \geqq 2$.

(c) $\operatorname{Pic}(Y)=Z$, generated by $\mathcal{O}_{Y}(1)$, if $r \geqq 3$.

These theorems are usually attributed to Lefschetz [26], although some cases were known earlier. The best modern proofs (in my opinion) are the ones of Andreotti and Frankel [1] and Bott [7] using Morse theory for (a), and the ones of Grothendieck [11] for (b) and (c). See Hartshorne [12, Chapter IV] for a simplified version of Grothendieck's proof, and for more detailed historical notes.

The principal Barth type theorems are the following.

THEOREM 2.2. Let $Y$ be a nonsingular subvariety of dimension $r$ of $\boldsymbol{P}_{\boldsymbol{C}}^{n}$ (which is not necessarily a complete intersection!). Then:

(a) The restriction map $H^{i}\left(\boldsymbol{P}^{n}, \boldsymbol{C}\right) \rightarrow H^{i}(Y, \boldsymbol{C})$ is an isomorphism for $i \leqq 2 r-n$.

(b) Ditto for cohomology with $\boldsymbol{Z}$ coefficients.

(c) $\pi_{1}(Y)=1$ if $r \geqq \frac{1}{2}(n+1)$.

(d) $\operatorname{Pic}(Y)=Z$, generated by $\mathcal{O}_{Y}(1)$, if $r \geqq \frac{1}{2}(n+2)$.

Of course (a) is a consequence of (b), but we state them separately for historical reasons. Part (a) was first proved by Barth [4], using the cohomology of coherent analytic sheaves on $\boldsymbol{P}^{n}$. Part (c) was proved by Barth and Larsen [5]. Part (b) was proved by Larsen [25], using Morse theory in addition to Barth's earlier techniques. Larsen also has statements about higher homotopy groups. Part (d) can be deduced from (b) using the exponential sequence. A more algebraic proof of (a) was given by Hartshorne [13]. Then purely algebraic proofs of (a), (c) for the algebraic fundamental group, and (d), using algebraic De Rham cohomology, were given by Ogus [30]. In characteristic $p>0$, there are analogous results using $Z / p Z$ cohomology (see [13] and [17]).

Note in the statements of the theorem that the restrictions on $i$ and $r$ operate so that if $r<\frac{1}{2} n$ there is no conclusion. On the other hand, as the codimension of $Y$ becomes small with respect to the dimension of $Y$, one has progressively stronger conclusions.

Now I would like to show how Theorem 2.2a is an easy consequence of the strong Lefschetz theorem. First recall

Theorem 2.3 (Strong LefsChetz theorem). Let $X$ be a nonsingular projective algebraic variety of dimension $n$, and let $\xi \in H^{2}(X, C)$ be the class of a hyperplane section. Then the cup-product map $H^{i}(X, C) \rightarrow^{\xi^{n-i}} H^{2 n-i}(X, C)$ is an isomorphism, for each $i=0,1, \cdots, n$.

This theorem is proved using Hodge's theory of harmonic integrals (see e.g. Weil [39]). 
COROLLARY 2.4. With the hypotheses of the theorem, the cup-product map $H^{i}(X, C) \rightarrow{ }^{\xi \jmath} H^{i+2 j}(X, C)$ is injective whenever $j \leqq n-i$.

Proof of 2.2a. Let $Y$ be a nonsingular subvariety of dimension $r$ of $P=\boldsymbol{P}_{C}^{n}$, and let $j: Y \rightarrow P$ be the inclusion map. Let $j^{*}: H^{i}(P) \rightarrow H^{\prime}(Y)$ be the restriction map on cohomology (always with $C$ coefficients), and let $j_{*}: H^{\imath}(Y) \rightarrow H^{i+2 n-2 r}(P)$ be the covariant map induced by Poincaré duality from the covariant map $j_{*}$ on homology. Let $\eta \in H^{2 n-2 r}(P)$ be the cohomology class of $Y$. Then for any $x \in H^{2}(P)$, we have $j_{*} j^{*}(x)=x \cup \eta$ in $H^{i+2 n-2 r}(P)$. This follows from the projection formula. On the other hand, for any $y \in H^{i}(Y)$, we have $j^{*} j_{*}(y)=y \cup j^{*}(\eta)$ in $H^{i+2 n-2 r}(Y)$. This follows from the Thom isomorphism theorem on a tubular neighborhood of $Y$.

Now suppose $Y$ is a variety of degree $d$. Then $\eta=d \cdot \xi^{n-r}$, where $\xi \in H^{2}(P)$ is the class of a hyperplane. Applying Corollary 2.4 to $Y$, we find that the map $j^{*} j_{*}: H^{i}(Y) \rightarrow H^{i+2 n-2 r}(Y)$, which is the cup-product with $j^{*}(\eta)=d \cdot j^{*}(\xi)^{n-r}$, is injective provided that $n-r \leqq r-i$, i.e., $i \leqq 2 r-n$. If this is so, then $j_{*}: H^{i}(Y) \rightarrow H^{2+2 n-2 r}(P)$ must also be injective. On the other hand, $j^{*} j_{*}: H^{i}(P) \rightarrow H^{i+2 n-2 r}(P)$ is an isomorphism. Indeed, these groups are either both 0 , if $i$ is odd, or both $C$, if $i$ is even, and they are generated by the appropriate powers of $\xi$, so the cup-product with $\xi^{n-r}$ is an isomorphism. Putting these facts together, we find that $j^{*}: H^{i}(P) \rightarrow H^{\imath}(Y)$ is an isomorphism, provided $i \leqq 2 r-n$.

REMARKS. 1. This argument can be applied to other ambient varieties besides projective space. For example, suppose $X$ is a nonsingular complete intersection variety of dimension $n$, and $Y$ is a nonsingular subvariety of dimension $r$. By (2.1) we know the cohomology of $X$. The same argument works, and we find that $j^{*}: H^{i}(X) \rightarrow H^{i}(Y)$ is an isomorphism for $i<2 r-n$, and injective for $i=2 r-n$.

2. Since the strong Lefschetz theorem has recently been proved by Deligne [9] for $l$-adic cohomology of projective varieties in characteristic $p$, and since the properties of $j_{*}$ and $j^{*}$ we used are formal properties which hold in any good cohomology theory, we conclude that the first Barth Theorem 2.2a holds also for the $l$-adic cohomology in characteristic $p$, provided $(l, p)=1$.

3. Examples. Subvarieties of small degree. Coming back to the original conjecture, let us make a few elementary observations. First of all, any hypersurface is a complete intersection, by definition. In codimension $\geqq 2$, however, there are varieties which are not complete intersections. The simplest example is the twisted cubic curve in $\boldsymbol{P}^{3}$, whose affine part is given parametrically by $x=t, y=t^{2}, z=t^{3}$. This is a curve of degree 3 , which does not lie in any plane. If it were the transversal intersection $H \cap H^{\prime}$ of two surfaces of degrees $d$ and $e$, then Bézout's theorem tells us 
the curve would have degree $d e$. But if $d e=3$ then $d$ or $e$ must be 1 , so $H$ or $H^{\prime}$ is a plane, which is impossible.

By taking the cone in $\boldsymbol{P}^{n}$ with vertex $\boldsymbol{P}^{n-4}$ over the twisted cubic curve in $\boldsymbol{P}^{3}$, one can construct a (singular) subvariety of $\boldsymbol{P}^{n}$, of codimension 2 , which is not a complete intersection. Hence the nonsingularity of $Y$ is an essential condition in our conjecture.

At present there are only vague heuristic reasons for the fraction $\frac{2}{3}$ which occurs in the conjecture. But at least we can show by example that it is the best possible. Let $G=G(1,4)$ denote the Grassmann variety of all projective lines in a projective 4-space. Then $G$ is a variety of dimension 6, which has a natural embedding (its Plücker embedding) in $\boldsymbol{P}^{9}$, and in this embedding it has degree 5 . Now $G$ is not contained in any hyperplane $\boldsymbol{P}^{8}$, so by Bézout's theorem as above it cannot be a complete intersection. (Another reason why $G$ is not a complete intersection is that $H^{4}(G, C)=C \oplus C$, which would contradict Lefschetz' Theorem 2.1a.)

This is rather meager evidence for the correctness of the fraction $\frac{2}{3}$. There are surfaces in $\boldsymbol{P}^{4}$ and 3 -folds in $\boldsymbol{P}^{5}$ which are not complete intersections. I do not know if there is a 4 -fold in $\boldsymbol{P}^{6}$ which is not a complete intersection. The first unknown case to which the conjecture applies is that of 5-folds in $\boldsymbol{P}^{7}$. For large values of $n$, it is easy to find noncomplete intersections of dimension approximately $\frac{1}{2} n$ in $\boldsymbol{P}^{n}$. But I do not know any infinite sequences of examples of noncomplete intersections which would justify the fraction $\frac{2}{3}$ of the conjecture as $n \rightarrow \infty$.

One way we can approach the conjecture is to study varieties according to their degree, instead of their dimension. A variety of degree 1 is a linear variety, which is itself a projective space, hence a complete intersection. If $Y$ is a variety of degree 2 and dimension $r$ in $\boldsymbol{P}^{n}$, then in fact $Y$ is contained in some $\boldsymbol{P}^{r+1}$. Hence it is a hypersurface in $\boldsymbol{P}^{r+1}$, and as such it is a complete intersection. More generally, one can show by an elementary argument that a variety $Y$ of dimension $r$ and degree $d$ in some $\boldsymbol{P}^{n}$ is always contained in a linear space $\boldsymbol{P}^{r+d-1}$.

For varieties of degree 3 , we have the following result, published anonymously [40]. The proof is completely elementary.

THEOREM 3.1. Let $Y$ be a nonsingular variety of dimension $r$ and degree 3 in $\boldsymbol{P}^{n}$, and assume that $Y$ is not contained in any $\boldsymbol{P}^{n-1}$. Then either

(a) $r=n-1$, i.e. $Y$ is a hypersurface, or

(b) $r=n-2, n=3,4$, or 5, and $Y$ is obtained by linear automorphism of $\boldsymbol{P}^{n}$ from one of the following three varieties:

(b1) the Segre embedding of $\boldsymbol{P}^{\mathbf{1}} \times \boldsymbol{P}^{\mathbf{2}}$ in $\boldsymbol{P}^{\mathbf{5}}$,

(b2) its general hyperplane section, which is a rational ruled surface in $\boldsymbol{P}^{\mathbf{4}}$,

(b3) the twisted cubic curve in $\boldsymbol{P}^{3}$. 
In particular, we see that if $Y$ is a nonsingular variety of degree 3 which is not a complete intersection, then it is contained in a projective space of dimension $n \leqq 5$.

A similar but more complicated analysis was carried out for varieties of degree 4 by Swinnerton-Dyer [37]. His result is this.

THEOREM 3.2. Let $Y$ be a nonsingular variety of dimension $r$ and degree 4 in $\boldsymbol{P}^{n}$, which is not contained in any $\boldsymbol{P}^{n-1}$. Then either

(a) $r=n-1$, so $Y$ is a hypersurface, or

(b) $r=n-2$, and $Y$ is a complete intersection of two quadric hypersurfaces, or

(c) $n \leqq 7$, and $Y$ is one of the following (up to an automorphism of $\boldsymbol{P}^{n}$ ):

(c1) the Segre embedding of $\boldsymbol{P}^{\mathbf{1}} \times \boldsymbol{P}^{\mathbf{3}}$ in $\boldsymbol{P}^{\mathbf{7}}$,

(c2) the Veronese embedding of $\boldsymbol{P}^{2}$ in $\boldsymbol{P}^{5}$,

(c3) a variety obtained from (c1) or (c2) by a succession of sections by a hyperplane and/or projections from a point into a lower-dimensional projective space.

As in the case of degree 3 , we see that those varieties of degree 4 which are not complete intersections are contained in a projective space of bounded dimension, in this case $n \leqq 7$, and that there is a finite list of possibilities. Unfortunately this method of complete classification is hopeless to extend to the general case. Already for degree 5 the calculations appear formidable.

However, using different techniques, one can show the existence of such a bound for any $d$. I understand that Barth and van de Ven [6] have also obtained a similar result by different methods. The result is this.

THEOREM 3.3. Given $d>0$, there exists an $n_{0}>0$, such that if $Y$ is a nonsingular projective variety of degree $d$, defined over $C$, which is not a complete intersection, then $Y$ is contained in some $\boldsymbol{P}^{n}$ with $n \leqq n_{0}$. Furthermore, there is only a finite number of continuous families of such varieties.

The proof of this result will appear in [15]. Let me just give a rough idea of what it depends on. If the result were false, one could find an infinite sequence of varieties $Y_{n} \subseteq \boldsymbol{P}^{n}$ of degree $d$, for each $n$. Moreover, we may assume that $Y_{n} \cap \boldsymbol{P}^{n-1}=Y_{n-1}$ for each $n$. Then, following an idea of Schwarzenberger (see [20, p. 165]), one can use Riemann-Roch and some analytic number theory to show that the Chern classes of the normal bundle of $Y$, and hence also the Hilbert polynomial of $Y$, are the same as those of a complete intersection. Then one uses the Barth-type Theorem $2.2 \mathrm{~d}$ above, the Kodaira vanishing theorem, and Riemann-Roch again, to show that the ideal of $Y$ is generated by the right number of elements. Unfortunately, because of the use of the Chebotarev density theorem 
in the first part of the proof, it does not seem possible by this method to find an effective bound for $n_{0}$ in terms of $d$. On the other hand, the method of Barth and van de Ven does give an effective bound, but it is limited so far to the case of codimension $2 .^{2}$

4. Embedding varieties in projective space. Let us approach our question from another angle. Given an algebraic variety $Y$ of dimension $r$, we can ask, what is the smallest $n$ such that there exists an embedding of $Y$ in $\boldsymbol{P}^{n}$ ? Of course there are abstract algebraic varieties which do not admit an embedding in any projective space (see Nagata [29] and Hironaka [18]), so let us restrict our attention to projective varieties, i.e. those which do admit some projective embedding.

Given an embedding of $Y$ in $\boldsymbol{P}^{n}$, we can define a mapping of $Y$ into $\boldsymbol{P}^{n-1}$ by projecting from a point of $\boldsymbol{P}^{n}$ to a hyperplane. One can show easily that if $Y$ is nonsingular, and if $n>2 r+1$, where $r=\operatorname{dim} Y$, then the projection can be chosen so that the image is still nonsingular, and hence we obtain an embedding of $Y$ in $\boldsymbol{P}^{n-1}$. Indeed, we need only make sure that the center $P$ of the projection map does not lie on any chord, or any tangent line of $Y$. By counting constants, we see that the chord variety of $Y$, which is the locus of all points on chords and tangents of $Y$, has dimension $\leqq 2 r+1$. So if $n>2 r+1$, we can find a point in $\boldsymbol{P}^{n}$ not on the chord variety.

Thus by successive projection we show that a nonsingular projective variety $Y$ of dimension $r$ can always be embedded in $\boldsymbol{P}^{2 r+1}$. This argument has been generalized by Lluis [27], who considers also singular varieties. He obtains an expression for the least expected embedding dimension which depends also on the singularities of $Y$.

In general, if we attempt to project $Y$ further into smaller projective spaces, the image will acquire singularities. See Roberts [32] for a study of what kind of singularities can occur.

However, in special cases, $Y$ may already lie in a smaller projective space, or the projection of $Y$ into a smaller projective space may remain nonsingular. So we can ask when does this happen? Let us consider some examples.

If $Y$ is a nonsingular curve, then $Y$ can always be embedded in $\boldsymbol{P}^{\mathbf{3}}$. But, unless it already lies in $\boldsymbol{P}^{\mathbf{2}}$, its generic projection into $\boldsymbol{P}^{\mathbf{2}}$ will always be singular. Another way of saying this is that a nonsingular plane curve cannot be the projection of a nonplanar curve in $\boldsymbol{P}^{3}$. The reason is that the linear system of line sections of a curve in $\boldsymbol{P}^{2}$ is a complete linear system

${ }^{2}$ Added in proof. Barth and van de Ven have extended their proof to treat all codimensions. On the other hand, my proof has developed some difficulties, and works at present only for codimension $\leqq 4$. 
of dimension 2. If the curve were the projection of a space curve, this linear system would be a subsystem of the linear system of plane sections of the space curve, which would have dimension 3 , so the original linear system would not be complete.

More generally, if $Y$ is a nonsingular subvariety of $\boldsymbol{P}^{n}$, then $Y$ can be realized as the projection of a variety in $\boldsymbol{P}^{n+1}$, not lying in any $\boldsymbol{P}^{n}$, if and only if the linear system of hyperplane sections is not complete. See Samuel [33, p. 27] for a more thorough discussion of this point. If the linear system of hyperplane sections is complete, we will say that $Y$ is linearly normal for the given embedding. In terms of sheaf cohomology, this says that the natural map $H^{0}\left(\boldsymbol{P}^{n}, \mathcal{O}_{\boldsymbol{P}}(1)\right) \rightarrow H^{0}\left(Y, \mathcal{O}_{Y}(1)\right)$ is surjective.

Being linearly normal is weaker than the condition of being projectively normal, which says that for all integers $k \in Z, H^{0}\left(\boldsymbol{P}^{n}, \mathcal{O}_{\boldsymbol{P}}(k)\right) \rightarrow H^{0}\left(Y, \mathcal{O}_{Y}(k)\right)$ is surjective. $Y$ is projectively normal if and only if the vertex of the cone over $Y$ in $\boldsymbol{A}^{n+1}$ is a normal point.

Coming back to complete intersections, one knows that any nonsingular complete intersection variety is projectively normal. Hence it is linearly normal, and so cannot be realized as a projection of a variety sitting effectively in a higher-dimensional projective space. This generalizes the example of plane curves above.

Now let us look at some more examples. A nonsingular surface in $\boldsymbol{P}^{3}$ is projectively normal, hence cannot be a projection of a surface in any higher $\boldsymbol{P}^{n}$. But a surface in $\boldsymbol{P}^{4}$ might be a projection of a surface in $\boldsymbol{P}^{5}$. So let us consider a nonsingular surface $Y$ in $\boldsymbol{P}^{5}$, which does not lie in any $\boldsymbol{P}^{\mathbf{4}}$. In general, its generic projection into $\boldsymbol{P}^{\mathbf{4}}$ will have a finite number of double points with transversal tangent planes. There are classical formulae for finding the number of these double points in terms of the numerical invariants of $Y$ and its embedding (see Semple and Roth [34, p. 196]). There is a remarkable theorem of Severi [36] which tells us when the projection is nonsingular.

THEOREM 4.1 (SEVERI). Let $Y$ be a surface in $\boldsymbol{P}^{5}$, not contained in any hyperplane, whose generic projection into $\boldsymbol{P}^{4}$ is nonsingular. Then, up to an automorphism of $\boldsymbol{P}^{5}, Y$ is the Veronese surface, which is the embedding of $\boldsymbol{P}^{2}$ in $\boldsymbol{P}^{5}$ given by

$$
\left(x_{0}, x_{1}, x_{2}\right) \mapsto\left(x_{0}^{2}, x_{0} x_{1}, x_{1}^{2}, x_{0} x_{2}, x_{1} x_{2}, x_{2}^{2}\right) .
$$

In higher dimensions, we know a few interesting examples of varieties which can be projected down into smaller projective spaces than one would expect. The example of the Veronese surface can be generalized as follows. Embed $\boldsymbol{P}^{r}$ in $\boldsymbol{P}^{N}$ by the 2-uple embedding, where $N=\frac{1}{2} r(r+3)$. Let $Y$ be its generic projection into $\boldsymbol{P}^{2 r+1}$. Then in this case $Y$ can be projected into $\boldsymbol{P}^{2 r}$ and remains nonsingular. The reason is that any two 
points of $Y$ (which is isomorphic to $\boldsymbol{P}^{r}$ ) can be joined by a line in $\boldsymbol{P}^{r}$, which becomes a conic in the given embedding of $Y$ in $\boldsymbol{P}^{2 r+1}$. Hence every point of the chord variety of $Y$ must lie on infinitely many chords of $Y$. So the dimension of the chord variety is $\leqq 2 r$, which allows us to project once more without acquiring singularities.

By a similar argument, one can show that the Segre embedding of $\boldsymbol{P}^{r} \times \boldsymbol{P}^{s}$ in $\boldsymbol{P}^{N}$ can be projected down to $\boldsymbol{P}^{2(r+s)-1}$ without acquiring singularities. This example, which was suggested by Tango, is in a space of two dimensions less than one would expect.

A third example of a similar nature is given by the Grassmann variety $G(1, m)$ of projective lines in $\boldsymbol{P}^{m}$. This is a variety of dimension $2(m-1)$, which has a natural embedding in a large projective space $\boldsymbol{P}^{n}$, and it can be projected down into $\boldsymbol{P}^{\mathbf{4} m-7}$ without acquiring singularities. This is four dimensions less than the general case,

The general philosophy suggested by Severi's theorem is that these events should be rather rare. More specifically, looking at the examples of the Veronese surface in $\boldsymbol{P}^{4}, \boldsymbol{P}^{2} \times \boldsymbol{P}^{2}$ in $\boldsymbol{P}^{7}$, and $G(1,5)$ in $\boldsymbol{P}^{13}$, each one of which is the projection of a variety lying effectively in a larger projective space, we have three examples of a variety $Y$ of dimension $\boldsymbol{r}$ in $\boldsymbol{P}^{n}$, with $r=\frac{2}{3}(n-1)$, which is not linearly normal. Since we do not know any examples where $r$ is any larger with respect to $n$, we make the following conjecture.

Conjecture 4.2. Let $Y$ be a nonsingular variety of dimension $r$ in $\boldsymbol{P}^{n}$, with $r>\frac{2}{3}(n-1)$. Then $Y$ is linearly normal.

Of course in settling this conjecture, it would be nice also to classify all nonlinearly normal varieties with $r=\frac{2}{3}(n-1)$, so as to have a satisfactory generalization of Severi's theorem.

As noted above, a complete intersection is always linearly normal, so this conjecture would be a consequence of our original conjecture, except for the case $r=\frac{2}{3} n$. My feeling is that this conjecture should be easier to establish than the original one.

5. Connections with local algebra. There has been some very interesting recent work in local algebra which is closely connected with our conjecture. To explain this, let us first state a local version of the conjecture. Given $Y$ in $\boldsymbol{P}^{n}$, we consider the cone over $Y$ in $\boldsymbol{A}^{n+1}$. Let $A$ be the local ring of the origin in $A^{n+1}$, and let $\mathfrak{p} \subseteq A$ be the prime ideal of the cone over $Y$. Then $Y$ is a complete intersection in $\boldsymbol{P}^{n}$ if and only if $A / \mathfrak{p}$ is a complete intersection in $A$, i.e. $\mathfrak{p}$ can be generated by exactly $n-r$ elements, where $r=\operatorname{dim} Y$. This motivates stating the following conjecture in local algebra, which is actually stronger than the original conjecture. (Note the changes in the definitions of $r$ and $n$ !) 
Conjecture 5.1. Let $A$ be a regular local ring of dimension $n$, let $\mathfrak{p} \subset A$ be a prime ideal, such that $A / \mathfrak{p}$ has an isolated singularity, let $r=\operatorname{dim} A / \mathfrak{p}$, and suppose that $r>\frac{1}{3}(2 n-1)$. Then $A / p$ is a complete intersection.

There are affirmative answers to this conjecture in the special cases of codimension 2 and 3, provided one is willing to make some auxiliary hypotheses on the depth of $A / \mathfrak{p}$. Here depth means the length of a maximal regular sequence in the maximal ideal. For example, depth $A / \mathfrak{p} \geqq 2$ would be equivalent to assuming that $A / p$ is normal, which corresponds in the global case to assuming $Y$ to be projectively normal.

I would like to mention briefly some of these results, and refer to the papers listed for more details and related results.

THEOREM 5.2 (SzPIRo [38]). Let $A$ be a regular local ring of dimension $n \geqq 7$, let $\mathfrak{p} \subseteq A$ be a prime ideal, such that $A / \mathfrak{p}$ is a locally complete intersection except at the closed point. Assume that $A / p$ is Cohen-Macaulay (i.e. $\operatorname{depth} A / \mathfrak{p}=\operatorname{dim} A / \mathfrak{p}$ ) and that it has dimension $r=n-2$. Then $A / \mathfrak{p}$ is a complete intersection.

The proof of this result uses a technique going back to Macaulay to show that $\mathfrak{p}$ is a determinantal ideal. Then from studying the singularities of determinantal loci, one obtains the result. See also Peskine and Szpiro [31] for related results.

The next result, also in codimension 2 , has a weaker depth hypothesis, but is proved only in characteristic 0 . It is due to Ogus and myself [14].

THEOREM 5.3. Let $A$ be a regular local ring containing its residue field $k$ of characteristic 0 . Let $\mathfrak{p} \subseteq A$ be a prime ideal such that $A / \mathfrak{p}$ is a complete intersection except at the closed point. Assume that $\operatorname{dim} A=n \geqq 7, \operatorname{dim} A / \mathfrak{p}=$ $r=n-2$, and that $\operatorname{depth} A / \mathfrak{p}>\frac{1}{2}(r+1)$. Then $A$ is a complete intersection.

The proof of this result uses a local analogue of the Barth-type theorem on Pic (Theorem 2.2d above), due to Ogus [30], and local duality, to show that $A / p$ is Gorenstein. Then the result follows from Serre's theorem [35] that Gorenstein in codimension 2 implies complete intersection.

THEOREM 5.4. With the hypotheses of Theorem 5.2, assume instead that $n \geqq 11, r=n-3$, and $A / \mathfrak{p}$ is Gorenstein. Then $A / \mathfrak{p}$ is a complete intersection.

This follows from work of Buchsbaum and Eisenbud [8]. They show that an ideal $\mathfrak{p}$ such that $A / \mathfrak{p}$ is Gorenstein of codimension 3 is generated by the Pfaffians of a certain skew symmetric matrix. Then a study of the singularities of such loci gives the result.

Using the techniques of Ogus and myself [14] as above, the Gorenstein hypothesis in Theorem 5.4 can be relaxed to depth $A / \mathfrak{p}>\frac{1}{2}(r+1)$ if we work in characteristic zero. 
It will be interesting to see if these techniques can be generalized somehow to higher codimensions.

6. Existence of vector bundles on $\boldsymbol{P}^{n}$. Closely related to the question whether subvarieties of small codimension of $\boldsymbol{P}^{n}$ are complete intersections, is the question whether there are vector bundles of small rank on $\boldsymbol{P}^{n}$, which are not isomorphic to a direct sum of line bundles. Let me first discuss the existence of vector bundles on $\boldsymbol{P}^{n}$, then explain the connection with the subvarieties.

In its crudest form, the question is for what integers $r$ does there exist an algebraic vector bundle $E$ of rank $r$ on $\boldsymbol{P}^{n}$ which is not a direct sum of line bundles. Or, equivalently, we can ask for what $r$ does there exist an indecomposable vector bundle of rank $\boldsymbol{r}$ on $\boldsymbol{P}^{n}$, where indecomposable means not a direct sum of bundles of smaller rank.

On $\boldsymbol{P}^{1}$, Grothendieck [10] has shown that every vector bundle is a direct sum of line bundles, so there is no problem.

On $\boldsymbol{P}^{n}$ for $n \geqq 2$, one knows there are indecomposable bundles of rank greater than 1. For example, the tangent bundle, which has rank $n$, is always indecomposable. More generally, Maruyama [28] has shown that for $n \geqq 2$ and $r \geqq n$, there exist indecomposable bundles of rank $r$ on $\boldsymbol{P}^{n}$. In fact, one can find algebraic families of mutually nonisomorphic ones, where the parameter variety has arbitrarily large dimension.

So we will restrict our attention to the range $2 \leqq r \leqq n-1$. In this range it is known that there exist bundles of rank $n-1$ on $\boldsymbol{P}^{n}$ for $n$ odd, which are not direct sums of line bundles, and that there exists at least one indecomposable bundle of rank 2 on $\boldsymbol{P}^{4}$ (see Horrocks and Mumford [23]). For $n \geqq 5$ and $2 \leqq r \leqq n-2$, it is not known whether there exist any indecomposable bundles at all.

If bundles do exist for certain values of $r$ and $n$, then we can ask further questions, such as what possible values can be taken on by the Chern classes of these bundles? The Riemann-Roch theorem imposes certain necessary conditions on the Chern classes of a vector bundle (see Hirzebruch [20, p. 166]). For example, if $E$ is a bundle of rank 2 on $\boldsymbol{P}^{3}$, then we must have $c_{1}(E) \cdot c_{2}(E) \equiv 0(\bmod 2)$. Horrocks [22] has shown by construction, that all possible values of $c_{1}$ and $c_{2}$ subject to this condition can occur as the Chern classes of a bundle of rank 2 on $\boldsymbol{P}^{3}$.

An interesting result of Schwarzenberger [20, p. 165] concerns the limiting situation with $r$ fixed, as $n$ tends to infinity. His result says that if $E$ is a bundle on $\boldsymbol{P}^{n}$, and if for every $n^{\prime}>n$ there exists a bundle $E^{\prime}$ on $\boldsymbol{P}^{n^{\prime}}$ of the same rank, such that $E^{\prime}$ restricted to $\boldsymbol{P}^{n}$ is $E$, then the Chern classes of $E$ are the same as the Chern classes of a suitable direct sum of line bundles. Recently Barth and van de Ven [6] have shown that if in addition $E$ has rank 2, then $E$ is a direct sum of line bundles. 
If we work over the complex numbers, then there are also the methods of algebraic topology available to study vector bundles. We forget the algebraic structure of $\boldsymbol{P}_{C}^{n}$, and consider it just as a differentiable manifold. Then for each $\boldsymbol{r}$ we can consider differentiable $\boldsymbol{C}^{r}$-bundles on $\boldsymbol{P}^{n}$. For $r \geqq n$, the "stable range", they are uniquely determined by their Chern classes. For $r<n$, the methods of homotopy theory are available (in principle) to achieve their complete classification. Then we can ask the question whether every differentiable $\boldsymbol{C}^{r}$-bundle has a holomorphic (hence also an algebraic) structure.

In this connection, Atiyah and Rees [3] have shown that for every $c_{1}, c_{2} \in Z$ with $c_{1} c_{2} \equiv 0(\bmod 2)$, there exists a differentiable $C^{2}$-bundle on $\boldsymbol{P}^{3}$. For $c_{1}$ odd, this bundle is unique, and for $c_{1}$ even there are exactly two nonisomorphic ones. In the case $c_{1}$ even there is a certain homotopy invariant which distinguishes the two. They find a way of expressing this invariant in algebraic terms, and this allows them to show, using the algebraic bundles constructed by Horrocks [22], that every differentiable $\boldsymbol{C}^{2}$-bundle on $\boldsymbol{P}^{3}$ has an algebraic structure.

On the other hand, Atiyah and Rees show that there exists a differentiable $\boldsymbol{C}^{2}$-bundle on $\boldsymbol{P}^{5}$ which has no algebraic structure.

Now let me explain the connection between the vector bundle question and the complete intersection question. Suppose $E$ is a bundle of rank $r$ on $\boldsymbol{P}^{n}$, with $r<n$. Then after tensoring $E$ with a suitable ample line bundle, if necessary, we can find a section $s \in \Gamma\left(\boldsymbol{P}^{n}, E\right)$ whose zero set $Y$ is an irreducible nonsingular subvariety of codimension $r$ of $\boldsymbol{P}^{n}$ [24, Corollary 3.6]. Furthermore, it is clear that if $E$ is a direct sum of line bundles, then $Y$ will be a complete intersection.

In the case of rank 2 we can say more.

Proposition 6.1. We work in $\boldsymbol{P}^{n}$, with $n \geqq 3$. A nonsingular subvariety $Y$ of codimension 2 in $\boldsymbol{P}^{n}$ occurs as the zero-set of a section of a bundle $E$ of rank 2 on $\boldsymbol{P}^{n}$ if and only if its canonical sheaf $\omega_{Y}$ is a multiple of the hyperplane sheaf $\mathcal{O}_{Y}(1)$. Furthermore, in that case, $E$ is unique, and $Y$ is a complete intersection if and only if $E$ is a direct sum of two line bundles.

Proof (Following Serre [35] and Horrocks [22]). If $Y$ is the zero-set of a section $s \in \Gamma(E)$, then we think of $s$ as a map from $\mathcal{O}_{P}$ to $E$. Taking duals, we get an exact sequence $0 \rightarrow L \rightarrow \breve{E} \rightarrow \mathcal{O}_{P} \rightarrow \mathcal{O}_{Y} \rightarrow 0$, where $L$ is a line bundle on $\boldsymbol{P}$. From this sequence we can calculate the canonical sheaf on $Y$ :

$$
\omega_{Y}=\mathscr{E}_{x} t_{\mathcal{O}_{P}}^{2}\left(\mathcal{O}_{Y}, \omega_{P}\right) \cong \check{L} \otimes \omega_{P} \otimes \mathcal{O}_{Y}
$$

Thus the canonical class on $Y$ is the restriction of a divisor class on $\boldsymbol{P}^{n}$, and so is some multiple of a hyperplane section, say $\omega_{Y}=\mathcal{O}_{Y}(k)$. 
In the other direction, given $Y$ with $\omega_{Y} \cong \mathcal{O}_{Y}(k)$, we can recover $\stackrel{\vee}{E}$ uniquely as an extension of $I_{Y}$, the ideal sheaf of $Y$, by $L$, where we take $L=\mathcal{O}_{P}(-k-n-1)$. The extension is determined by the element

$$
\xi \in \operatorname{Ext}_{\mathscr{O}_{P}}^{1}\left(I_{Y}, L\right) \cong \operatorname{Ext}_{\mathscr{O}_{P}}^{2}\left(\mathcal{O}_{Y}, L\right)
$$

corresponding to $1 \in H^{0}\left(\mathcal{O}_{Y}\right)$ under the natural isomorphism one obtains from the spectral sequence of local and global Ext. Since $E$ is uniquely determined by $Y$, the complete intersections correspond to the direct sums of line bundles.

COROLlaRY 6.2. There exists an indecomposable vector bundle of rank 2 on $\boldsymbol{P}^{n}, n \geqq 3$, if and only if there exists a nonsingular subvariety $Y$ of codimension 2 with $\omega_{Y} \cong \mathcal{O}_{Y}(k)$ for some $k \in Z$.

Furthermore, if we are working over $C$, and if $n \geqq 6$, then the Barthtype Theorem $2.2 \mathrm{~d}$ says that every line bundle on $Y$ is a multiple of $\mathcal{O}_{Y}(1)$, so the last condition is superfluous. So our original conjecture in the case of codimension two at least is equivalent to the following.

Conjecture 6.3. If $n \geqq 7$, there are no indecomposable vector bundles of rank 2 on $\boldsymbol{P}^{n}$.

I do not feel that I have sufficient evidence to formulate a conjecture about bundles of rank $>2$. There are a number of questions which come to mind, however. For example, it would be nice to find necessary and sufficient conditions for a nonsingular subvariety $Y$ of codimension 3 to be the zero-set of a section of some vector bundle $E$ of rank 3 . And in that case one would like to express the condition that $E$ be a direct sum of line bundles in terms of some properties of $Y$.

\section{REFERENCES}

1. A. Andreotti and T. Frankel, The Lefschetz theorem on hyperplane sections, Ann. of Math. (2) 69 (1959), 713-717.

2. M. F. Atiyah and F. Hirzebruch, Analytic cycles on complex manifolds, Topology 1 (1962), 25-45. MR 26 \#3091.

3. M. F. Atiyah and E. Rees (to appear).

4. W. Barth, Transplanting cohomology classes in complex-projective space, Amer. J. Math. 92 (1970), 951-967. MR 44 \#4239.

5. W. Barth and M. F. Larsen, On the homotopy groups of complex projective algebraic manifolds, Math. Scand. 30 (1972), 88-94.

6. W. Barth and A. van de Ven, $A$ decomposability criterion for algebraic 2-bundles on projective spaces, Invent. Math. (to appear).

7. R. Bott, On a theorem of Lefschetz, Michigan Math. J. 6 (1959), 211-216.

8. D. Buchsbaum and D. Eisenbud, Gorenstein ideals of height 3 (to appear).

9. P. Deligne, La conjecture de Weil. I, Inst. Hautes Études Sci. Publ. Math. No. 43 (1974), 273-307. 
10. A. Grothendieck, Sur la classification des fibrés holomorphes sur la sphère de Riemann, Amer. J. Math. 79 (1957), 121-138.

11. - Cohomologie locale des faisceaux cohérents et théorèmes de Lefschetz locaux et globaux (SGA 2), North-Holland, Amsterdam, 1968.

12. R. Hartshorne, Ample subvarieties of algebraic varieties, Lecture Notes in Math., vol. 156, Springer-Verlag, Berlin and New York, 1970. MR 44 \#211.

13. - Cohomology of non-complete algebraic varieties, Compositio Math. 23 (1971), 257-264. MR 46 \#1793.

14. R. Hartshorne and A. Ogus, On the factoriality of local rings of small embedding codimension, Comm. Algebra 1 (1974), 415-437.

15. R. Hartshorne, Projective varieties of small degree (to appear).

16. R. Hartshorne, E. Rees and E. Thomas, Non-smoothing of algebraic cycles on Grassmann varieties, Bull. Amer. Math. Soc. 80 (1974), 847-851.

17. R. Hartshorne and R. Speiser, Local cohomological dimension in characteristic $p$, (to appear).

18. H. Hironaka, On the theory of birational blowing-up, Thesis, Harvard University, Cambridge, Mass., 1960 (unpublished).

19. - Smoothing of algebraic cycles of small dimensions, Amer. J. Math. 90 (1968), 1-54. MR 37 \#210.

20. F. Hirzebruch, Topological methods in algebraic geometry, 3rd ed., Die Grundlehren der math. Wissenschaften, Band 131, Springer-Verlag, Berlin and New York, 1966. MR 34 \#2573.

21. W. V. D. Hodge, The topological invariants of algebraic varieties, Proc. Internat. Congress Math. (Cambridge, Mass., 1950), vol. I, Amer. Math. Soc., Providence, R.I., 1952, pp. 182-192. MR 13, 679.

22. G. Horrocks, A construction for locally free sheaves, Topology 7 (1968), 117-120. MR 37 \#2765.

23. G. Horrocks and D. Mumford, A rank 2 bundle on $\boldsymbol{P}^{4}$ with 15,000 symmetries, Topology 12 (1973), 63-81.

24. S. L. Kleiman, Geometry on Grassmannians and applications to splitting bundles and smoothing cycles, Inst. Hautes Études Sci. Publ. Math. No. 36 (1969), 281-297. MR 42 \#281.

25. M. E. Larsen, On the topology of complex projective manifolds, Invent. Math. 19 (1973), 251-260.

26. S. Lefschetz, On certain numerical invariants of algebraic varieties with applications to Abelian varieties, Trans. Amer. Math. Soc. 22 (1929), 327-482.

27. E. Lluis Riera, Sur l'immersion des variétés algébriques, Ann. of Math. (2) 62 (1955), 120-127. MR 17, 87.

28. M. Maruyama, On a family of algebraic vector bundles, Number Theory, Algebraic Geometry, and Commutative Algebra, Tokyo, 1973, pp. 95-146.

29. M. Nagata, Existence theorems for nonprojective complete algebraic varieties, Illinois J. Math. 2 (1958), 490-498. MR 20 \#3875.

30. A. Ogus, Local cohomological dimension of algebraic varieties, Ann. of Math. (2) 98 (1973), 327-365.

31. C. Peskine and L. Szpiro, Liaison des variétés algébriques. I, Invent. Math. (to appear)

32. J. Roberts, Generic projections of algebraic varieties, Amer. J. Math. 93 (1971), 191-214. MR 43 \#3263.

33. P. Samuel, Méthodes d'algèbre abstraite en géométrie algébrique, 2nd ed., Ergebnisse der Mathematik und ihrer Grenzgebiete, Band 4, Springer-Verlag, Berlin and New York, 1967. MR 35 \#4211. 
34. J. G. Semple and L. Roth, Introduction to algebraic geometry, Clarendon Press, Oxford, 1949. MR 11, 535.

35. J.-P. Serre, Sur les modules projectifs, Séminaire P. Dubreil, M.-L. DubreilJacotin et C. Pisot, 14ième année: 1960/61. Algèbre et théorie des nombres, fasc. 1, exposé 2, Faculté des Science de Paris, Secrétariat mathématique, Paris, 1963. MR 28 \#3911.

36. F. Severi, Intorno ai punti doppi impropri di una superficie generale dello spazio a quattro dimensioni, e a suoi punti tripli apparenti, Rend. Circ. Mat. Palermo 15 (1901), $33-51$.

37. H. P. F. Swinnerton-Dyer, An enumeration of all varieties of degree 4, Amer. J. Math. 95 (1973), 403-418.

38. L. Szpiro, Variétés de codimension 2 dans $\boldsymbol{P}^{n}$, Colloq. d'algèbre Rennes, 1972, exposé 15 .

39. A. Weil, Variétés Kähleriennes, Hermann, Paris, 1958.

40. XXX. Correspondence, Amer. J. Math. 79 (1957), 951-952. 